\title{
The Effects of an Uninterrupted Switch from Donepezil to Galantamine without Dose Titration on Behavioral and Psychological Symptoms of Dementia in Alzheimer's Disease
}

\author{
Shoichi Sasaki ${ }^{a}$ Yoshiharu Horie $^{b}$ \\ a Department of Neurology, Tokyo Women's Medical University, Tokyo, and 'b Division of \\ Biostatistics, Kitasato University Graduate School, Sagamihara, Japan
}

\section{Key Words}

Alzheimer's disease - Behavioral and psychological symptoms of dementia - Donepezil ·

Drug switching · Galantamine · Mild cognitive impairment

\section{Abstract}

Aims: To elucidate the efficacy of galantamine on cognition and behavioral and psychological symptoms of dementia (BPSD) in outpatients with mild cognitive impairment (MCI) and Alzheimer's disease (AD) who have switched from donepezil to galantamine. Materials and Methods: We performed an uninterrupted switch from donepezil to galantamine without a washout period or dose titration in 44 ambulatory outpatients with amnestic MCI $(n=12)$ or mild-to-moderate AD $(n=32)$. Three months after the switch, the efficacy of galantamine was evaluated with the Mini-Mental State Examination (MMSE), and the Neuropsychiatric Inventory (NPI) and NPI Brief Questionnaire Form (NPI-Q), respectively, using the Wilcoxon signedrank test. Results: NPI scores improved significantly on BPSD, especially on delusions, agitation and aberrant motor activity in AD patients $(p=0.027)$; improvement was remarkable in patients with moderate AD (MMSE score 10-19; $p=0.007$ ), while insignificant in those with $M C I$ (MMSE score $\geq 24 ; p=0.648$ ). The NPI-Q score also improved significantly regarding both the severity of the disease $(p=0.009)$ and caregiver distress $(p=0.012)$ in AD patients. MMSE scores hardly improved in either MCI $(p=0.394)$ or AD patients $(p=0.265)$. Conclusions: $A n$ uninterrupted switch from donepezil to galantamine could be a useful alternative treatment option for AD patients whose BPSD are unresponsive to donepezil, or whose caregivers are not satisfied with donepezil treatment. 


\section{Introduction}

In Alzheimer's disease (AD), besides disturbance of cognitive function, behavioral and psychological symptoms of dementia (BPSD) such as delusions, hallucinations, depression/dysphoria, anxiety, agitation/aggression, elation/euphoria, disinhibition, irritability/ lability, apathy/indifference, and aberrant motor activity are common, affecting approximately $90 \%$ of patients at some stage of the disease $[1,2]$. Patients with mild AD have psychiatric symptoms (delusions, hallucinations) and emotional symptoms (agitation, dysphoria, anxiety, irritability) as frequently as those with moderate or severe AD [3], and BPSD do not necessarily correlate with the severity of cognitive changes [4, 5]. Moreover, BPSD are a major cause of increased caregiver stress and burden [6] and are associated with the decision to institutionalize AD patients [7]. Early and adequate treatment can ameliorate BPSD and improve the quality of life, easing the burden for both the patients with dementia and their caregivers.

Galantamine was launched in Japan in March 2011, about 10 years later than donepezil, and is now established as one of the standard therapies for mild to moderate AD. Galantamine has allosteric modulating activity at the nicotinic acetylcholine receptors (nAChRs) in addition to acting as an acetylcholinesterase inhibitor (AChEI) [8], and shows improvement of behavioral and neuropsychiatric symptoms [9]. In this study, the efficacy of galantamine on the symptoms of cognition and BPSD was evaluated by an uninterrupted switch from donepezil to galantamine with no washout period or dose titration in amnestic mild cognitive impairment (MCI) and AD patients, whose BPSD were unresponsive to donepezil, or whose caregivers were not satisfied with donepezil treatment.

\section{Materials and Methods}

\section{Patients}

The enrolled subjects were ambulatory outpatients who had MCI or mild to moderate probable $\mathrm{AD}$ according to the criteria of the National Institute of Neurological and Communicative Disorders and the Alzheimer's Disease and Related Disorders Association (NINCDSADRDA) $[10,11]$. The age range of the patients was 62-95 years $(n=46$, mean 83.3 years) and $58.7 \%(\mathrm{n}=27)$ of the patients were female. Patients had been taking donepezil for $\geq 33$ months (994 \pm 711.7 days) on average and had a Mini-Mental State Examination (MMSE) score of $>10$. They showed firm radiological evidence of AD on MRI with a prominent atrophy of the hippocampi and a corresponding enlargement of the inferior horns of the lateral ventricles; on SPECT, they showed a reduction of blood flow in the parietal and temporal regions. Under the category of amnestic MCI patients, we included those who had an MMSE score of $\geq 24$ and a reduction of blood flow in the precuneus and posterior cingulate gyrus by SPECT (3D-SSP or eZIS) or PET, accompanied by hippocampal atrophy. Exclusion criteria for the present study were as follows: evidence of neurodegenerative diseases other than AD that may cause or contribute to dementia such as frontotemporal dementia and dementia with Lewy bodies; cognitive impairment resulting from cerebrovascular dementia; metabolic disturbance such as hepatic, renal, pulmonary and endocrine disturbances; vitamin deficiency; epilepsy, and psychiatric disease. Patients were eligible only if they had a history of cognitive decline that was gradual in onset and progressive over a period of $>7$ months, and were living with responsible caregivers. Written informed consent to the clinical investigation was obtained from the patients or their caregivers. Ultimately, a sample of 46 Japanese outpatients meeting NINCDS-ADRDA criteria for $\mathrm{MCI}$ and probable AD were selected as pertinent subjects in the routine clinical setting. 
Safety assessments throughout the study were comprised of physical examinations, vital signs, standard laboratory tests as needed, and monitoring for adverse gastrointestinal symptoms such as nausea, vomiting and diarrhea.

\section{Design}

An uninterrupted switch from donepezil to galantamine with no washout period or dose titration was performed in $\mathrm{MCI}$ and $\mathrm{AD}$ patients who had gained little or no benefit from donepezil, or whose caregivers were not satisfied with donepezil treatment. Treatment with donepezil $5 \mathrm{mg} /$ day, $8 \mathrm{mg} /$ day, or $10 \mathrm{mg} /$ day was switched to the equivalent galantamine dosages of $16 \mathrm{mg} /$ day, $20 \mathrm{mg} /$ day, or $24 \mathrm{mg} /$ day, respectively. Concurrent use of other AChEIs and additional anti-dementia drugs was not permitted. Changes of antidepressants or psychotropic medications such as anxiolytics, hypnotics, and neuroleptics within 6 months before the switch, and addition of such drugs were not permitted. MMSE scores and the Japanese version of the Neuropsychiatric Inventory (NPI) including the Caregiver Distress Scale and the Neuropsychiatric Inventory Brief Questionnaire Form (NPI-Q) [12] were evaluated at the time just before and 3 months after the switch from donepezil to galantamine. The NPI is an instrument to assess the frequency and severity of symptoms, and the caregiver distress in 10 behavioral domains (delusions, hallucinations, depression/dysphoria, anxiety, agitation/aggression, elation/ euphoria, disinhibition, irritability/lability, apathy/indifference, and aberrant motor activity) of BPSD occurring in patients with dementia [13]. The NPI is given as an interview questionnaire, and neuropsychiatric manifestations within each domain are rated by the caregiver in terms of both frequency (1-4) and severity (1-3), yielding a composite symptom domain score (frequency $\times$ severity). Caregiver distress is rated for each positive neuropsychiatric symptom domain by scores from 0 (no distress) to 5 (extreme distress). The NPI has demonstrated stagespecific trends in neuropsychiatric symptoms in AD patients [4]. The NPI-Q provides a brief, reliable, informant-based assessment of neuropsychiatric symptoms and associated caregiver distress that may be suitable for use in routine clinical practice [14]. It is given as a self-administered questionnaire, as opposed to an interview. Written instructions for completing the questionnaire form are included for symptom severity and caregiver distress ratings. The NPI-Q scores in 10 behavioral domains are each rated in terms of the severity of the disease as 0 (absent); 1 (mild); 2 (moderate), and 3 (severe). The total NPI-Q severity score represents the sum of individual symptom scores and ranges from 0 to 30 . The NPI-Q caregiver distress scores are rated from 0 (no distress) to 5 (very severe). The total NPI-Q distress score represents the sum of individual symptom scores and ranges from 0 to 50 . The Japanese version of the NPI and NPI-Q is sufficiently valid and as reliable as the original version [12].

\section{Statistical Analysis}

MMSE, NPI and NPI-Q scores just before the switch from donepezil to galantamine and 3 months later were compared using the Wilcoxon signed-rank test. The level of significance for all comparisons (type I error) was set at $\mathrm{p}<0.05$. Moreover, NPI and NPI-Q scores were evaluated in subgroups of patients categorized according to AD severity: MCI (MMSE score $\geq 24$ ), mild AD (MMSE score 20-23) and moderate AD (MMSE score 10-19) using the Wilcoxon signed-rank test.

\section{Results}

A total of 44 out of 46 patients (95.7\%) completed the evaluation at 3 months. Two patients discontinued participation, due in one case to overexcitement and in the other to severe anorexia as a result of dehydration from excessive summer heat, both of which were 
Table 1. The clinical and medication profiles of the enrolled patients

\begin{tabular}{llcc}
\hline & AD & MCI & AD + MCI \\
\hline Number of patients & 32 & 12 & 44 \\
Sex (m/f) & $11 / 21$ & $8 / 4$ & $19 / 25$ \\
Mean age, years & 84.5 & 79.8 & 83.2 \\
Disease duration, months & 50.7 & 34.5 & 46.3 \\
Mean dosing period of donepezil, days & 1,056 & 829.9 & 994.3 \\
Mean dosing period of galantamine, days & 94.6 & 97.4 & 95.3 \\
Mean administered dose of donepezil, mg/day & 5.7 & 6.2 & 5.8 \\
Mean administered dose of galantamine, mg/day & 17.2 & 17.3 & 17.2 \\
Number of patients taking memantine & 4 & 1 & 5 \\
Mean MMSE score & 16.7 & 27.4 & 19.9 \\
Mean NPI score & 18.1 & 11.2 & 16.2 \\
\hline
\end{tabular}

not associated with side effects. The clinical and medication profiles of the 44 enrolled patients prior to the switch from donepezil to galantamine are shown in table 1 . The mean age was 83.2 years $(n=44)$, and $56.8 \%(n=25)$ of the patients were female. The mean duration of galantamine treatment was 95.3 days. Among the 44 patients, 32 patients took galantamine doses of $16 \mathrm{mg} /$ day, 7 patients $20 \mathrm{mg} /$ day, and 5 patients $24 \mathrm{mg} /$ day. As for 5 patients to whom memantine had been administered in addition to donepezil for more than 6 months before the switch, the same dose of memantine was maintained after the switch to galantamine. Other concomitant classes of medication were antihypertensive agents (16 patients, $36.4 \%$ ), antithrombotic agents (10 patients, 22.7\%), hypolipidemic agents (8 patients, $18.2 \%$ ), antidiabetics ( 6 patients, $13.6 \%$ ), memantine (5 patients, 11.4\%), an anticoagulant (1 patient, 2.3\%) and quetiapine (1 patient, 2.3\%). The uninterrupted switch from donepezil to galantamine was well tolerated in all patients and was not associated with cholinergic side effects.

In the AD group ( $\mathrm{n}=32$, MMSE score 10-23), the mean MMSE score showed no significant benefit from the switch $(17.1 \pm 3.42$ to $17.6 \pm 3.57, \mathrm{p}=0.265)$, while the mean NPI score exhibited statistically significant improvement at 3 months after the switch $(18.1 \pm 13.77$ to $14.7 \pm 11.0, \mathrm{p}=0.027$ ) compared with that just before the switch (fig. 1 ). In the MCI group ( $\mathrm{n}=12$, MMSE score $\geq 24$ ), there was no significant beneficial effect either on MMSE (27.4 \pm 2.02 to $27.1 \pm 2.93, \mathrm{p}=0.394)$ or NPI $(11.2 \pm 12.1$ to $10.8 \pm 12.81, \mathrm{p}=0.648)$ scores. Moreover, when separating patients into two groups, i.e., the mild group with $\mathrm{MCI}+$ mild $\mathrm{AD}(\mathrm{n}=20$, MMSE score $\geq 20$ ) and the moderate group with moderately advanced AD ( $n=24$, MMSE score 10-19), the significant beneficial effect of galantamine on the NPI score in the moderate group (MMSE score 10-19) 3 months after the switch (16.3 \pm 16.93 to $13.1 \pm 13.31, p=0.007)$ contrasted remarkably with the insignificant benefit in the mild group (MMSE score $\geq 20$; $16.1 \pm 16.93$ to $14.3 \pm 14.31, \mathrm{p}=0.39$ ) (fig. 2 ). Among the NPI subdomains, delusions ( $\mathrm{p}=$ $0.018)$, agitation $(p=0.034)$ and aberrant motor activity $(p=0.045)$ were significantly improved in AD patients (fig. 3).

The average NPI-Q score was significantly improved in terms of both the severity of the disease ( $6.2 \pm 4.83$ to $4.9 \pm 3.78, \mathrm{p}=0.009$ ) (fig. 4 ) and the caregiver distress $(6.7 \pm 5.74$ to 5.3 $\pm 4.42, \mathrm{p}=0.012$ ) (fig. 5) in $\mathrm{AD}$ patients, whereas in MCI patients there was no significant efficacy in disease severity ( $4.2 \pm 3.59$ to $4.3 \pm 4.01, \mathrm{p}=0.674)$ or distress burden $(4.3 \pm 4.35$ to $4.8 \pm 5.22, p=0.317$ ). The significant beneficial effect of galantamine on the NPI-Q score for disease severity $(5.7 \pm 3.85$ to $4.4 \pm 3.44, \mathrm{p}=0.001)$ and distress burden $(6.0 \pm 4.77$ to 4.6 $\pm 3.88, \mathrm{p}=0.004$ ) in the moderate group (MMSE score 10-19) contrasted remarkably with 
Fig. 1. Change of NPI score after 3 months in $\mathrm{AD}$ patients (mean \pm SD; $\mathrm{n}=32, \mathrm{p}=0.0027$ ).

Fig. 2. Change of NPI score after 3 months in moderately advanced AD (MMSE score 10-19; mean \pm $\mathrm{SD} ; \mathrm{n}=24, \mathrm{p}=0.007$ ).

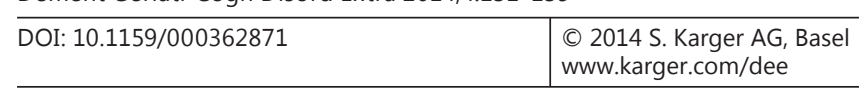

Sasaki and Horie: Effects of Galantamine on BPSD in AD
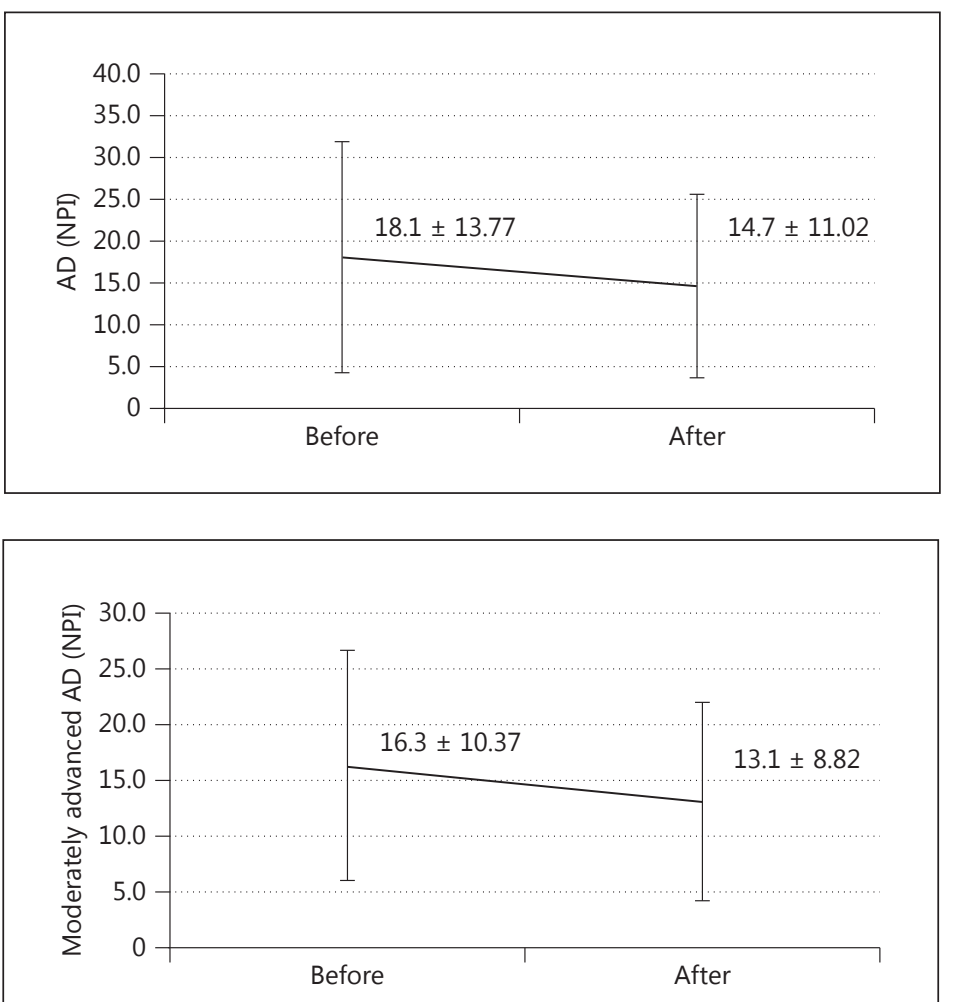

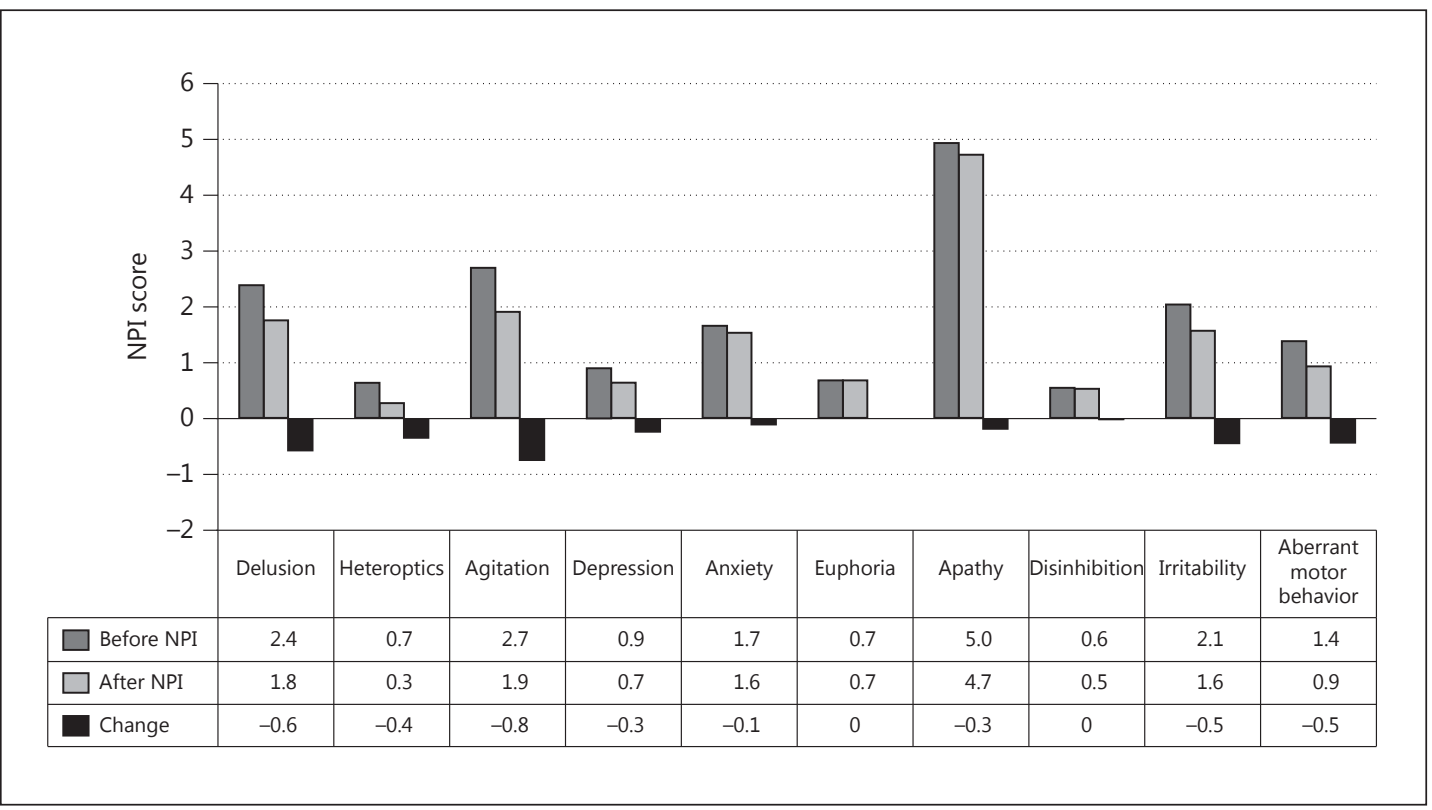

Fig. 3. Change of NPI score after 3 months in the subdomain of AD patients. Delusion: $p=0.018$; agitation: $\mathrm{p}=0.034 ;$ aberrant motor behavior: $\mathrm{p}=0.045$. 
Fig. 4. Change of NPI-Q subscore for severity of symptoms after 3 months in $\mathrm{AD}$ patients (mean \pm $\mathrm{SD} ; \mathrm{n}=32, \mathrm{p}=0.009$ ).

Fig. 5. Change of NPI-Q subscore for caregiver distress after 3 months in $\mathrm{AD}$ patients (mean \pm $\mathrm{SD} ; \mathrm{n}=32, \mathrm{p}=0.012$ ).
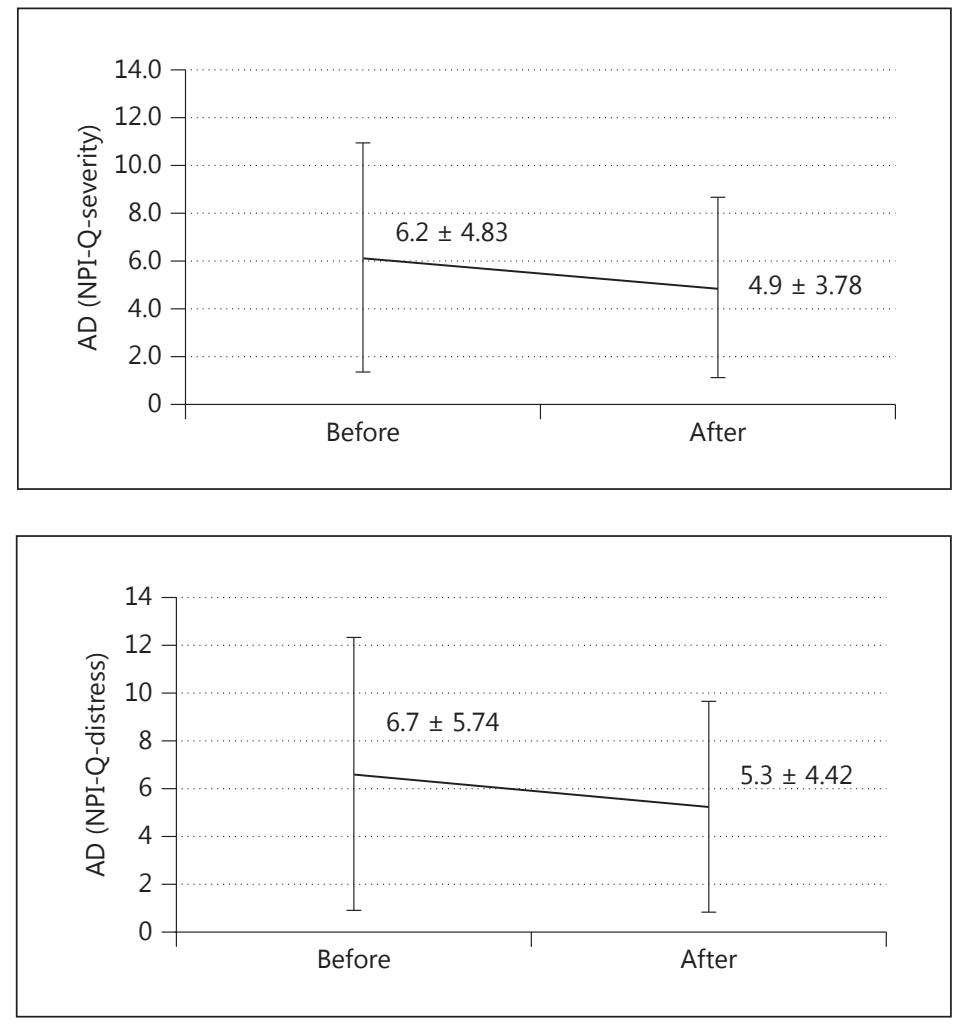

the insignificant benefit regarding severity (mean of difference $5.6 \pm 5.42$ to $5.15 \pm 4.26, \mathrm{p}=$ $0.562)$ and distress burden $(6.0 \pm 6.34$ to $5.8 \pm 5.35, \mathrm{p}=0.795)$ in the mild group (MMSE score $\geq 20$ ).

\section{Discussion}

BPSD or non-cognitive symptoms are very common in AD patients $[4,15]$ and can be assessed by standardized instruments such as NPI [4, 13]. In patients with AD, cognitive impairment and BPSD are correlated [15]. Moreover, BPSD give rise to noticeable disability in AD patients, an increase in caregiver distress [16], and poorer patient quality of life [17]. Worsening BPSD represent the main cause of earlier patient institutionalization (earlier nursing home entry) [7]. Therefore, early detection of BPSD and efficient treatment with medication is very important in delaying nursing home placement and improving both patients' and caregivers' quality of life.

Cholinergic deficiency has been associated with neuropsychiatric symptoms of AD as well as cognitive disturbance $[4,18]$; hence, it is logical to use AChEIs in the treatment of BPSD. According to an analysis of four trials on galantamine and behavior in AD, 5-6 months of galantamine treatment reduced behavioral symptoms of $\mathrm{AD}$ and the associated caregiver burden; the beneficial effects on the NPI were reported for galantamine versus a placebo [9, 19-21]. Moreover, several clinical trials involving galantamine in patients with mild to moderate AD showed improvement of neuropsychiatric symptoms and the associated caregiver distress [19, 22, 23]. Post hoc analysis of pooled data from three large trials of 3-, 5-, and 6 -month galantamine treatment for problematic behavior in $\mathrm{AD}$ patients showed a slightly 
significant benefit of galantamine over the placebo on total NPI scores, and in the domains of agitation/aggression, anxiety, disinhibition, and aberrant motor behavior [24]. In contrast, in another study, the beneficial effect of galantamine on the NPI scores after 3 months of treatment was not statistically significant, likely owing to the short duration of the trial and the exclusion of patients with behavioral problems at baseline [9, 25].

The present study showed that AD patients who switched from donepezil to the equivalent dosage of galantamine maintained the same cognitive function on MMSE 3 months after the switch, and the NPI scores in AD patients, in particular the scores in moderately advanced AD patients (MMSE score 10-19), improved significantly, while those in $\mathrm{MCI}+$ mild AD patients (MMSE score $\geq 20$ ) did not, which is consistent with that of a previous report [9]; among the subdomains, delusions, agitation and aberrant motor activity were significantly improved. Moreover, the significant beneficial effect of galantamine on the NPI-Q score, which is evaluated by a questionnaire through the caregiver, indicates that the caregivers of patients with AD experienced a significant benefit after the switch from donepezil while those of patients with MCI did not. Thus, the 3 months' duration of galantamine treatment after an uninterrupted switch from donepezil seems to be a long enough period to demonstrate the therapeutic beneficial effect on the NPI and NPI-Q.

Expression levels of nAChRs are reduced in the brains of AD patients, as compared with age-matched controls [26]. nAChRs are located on presynaptic membranes regulating the release of ACh and other neurotransmitters, including glutamate, GABA, 5-HT, and dopamine, via $\mathrm{Ca}^{2+}$ influx [27]. Anticholinesterase therapy for $\mathrm{AD}$ has in common the ability to inhibit cholinesterases [28]. Inhibition of cholinesterases increases the synaptic concentration of $\mathrm{ACh}$, thereby enhancing and prolonging the action of ACh on nicotinic receptors and on muscarinic receptors, leading to improvement of cognitive function. Galantamine binds to most nAChRs, and induces the release of neurotransmitters such as ACh, dopamine, noradrenaline, 5-HT, and GABA by nicotinic allosteric potentiating ligand action [29]. Thus, galantamine potentiates the action of ACh on nAChRs and does not alter the activity of nAChRs in the absence of $\mathrm{ACh}$; hence, nicotinic allosteric potentiating ligand action may contribute to the therapeutic effectiveness of galantamine in AD [8]. Improvement of the NPI and NPI-Q scores in this study may be due to a nicotinic allosteric potentiating ligand action on AChRs.

Regarding the washout period for the switch from donepezil to galantamine, there have been a variety of reports [30-32]. For example, there is no evidence of a loss of symptomatic benefit or changes in cognitive performance with either a 4- or 7-day washout period, and there are fewer gastrointestinal adverse events if the washout period is 4 days rather than 7 days [30]. A switch without a washout period and subsequent dose escalation (8-24 mg/day) over 3 weeks results in an increase in gastrointestinal adverse events, while either fast (weekly increments of $8 \mathrm{mg} /$ day) or slow (monthly increments of $8 \mathrm{mg} /$ day) galantamine dose titration after a 7-day donepezil washout period is well tolerated [31]. In contrast, AD patients switching from donepezil to galantamine without a washout period were reported to tolerate the switch well, with stabilization or improvement in cognitive function, behavior, and activities of daily living in the subsequent 3 months [32], although the number of patients examined was very few $(n=4)$. The peak levels of AChE inhibition achieved by the combined effect of donepezil and galantamine were above the level of inhibition achieved by donepezil alone for less than $1 \mathrm{~h}$, suggesting that the same dose escalation profile (starting from $4 \mathrm{mg}$ b.i.d.) that is used for first-time galantamine recipients could be used for patients who previously took donepezil [33]. Thus, given the tolerability and pharmacokinetic properties of galantamine, there is a pharmacokinetic rationale for switching from donepezil to galantamine without a washout period [33]. In fact, the uninterrupted switch from donepezil to the equivalent dosage of galantamine without dose titration in this study was well tolerated and not associated with cholinergic side effects, although galantamine is commonly associated 
with adverse events such as nausea (17.3\%), dizziness (12\%), and vomiting (10.7\%) [34]. The therapeutic strategy of no washout period and no dose titration may also be desirable because it provides treatment continuity for patients and continuous cholinergic stimulation.

These findings suggest that an uninterrupted switch from donepezil to the equivalent dosage of galantamine with no washout period or dose titration could be a useful alternative treatment option in moderate AD patients, whose BPSD are unresponsive to donepezil, or whose caregivers are not satisfied with donepezil treatment. This preliminary study indicates that a larger clinical trial is warranted to determine the efficacy of galantamine for BPSD.

\section{Acknowledgements}

The authors would like to thank the patients and their caregivers who took part in the study and Dr. S. Shimizu (Medical Research Institute, Tokyo Women's Medical University, Tokyo) for statistical analysis.

\section{Disclosure Statement}

The authors report no conflicts of interest.

\section{References}

1 Rovner BW, Kafonek S, Filipp L, Lucas MJ, Folstein MF: Prevalence of mental illness in a community nursing home. Am J Psychiatry1986;143:1446-1449.

2 Tariot PN, Podgorski CA, Blazina L, Leibovici A: Mental disorders in the nursing home: another perspective. Am J Psychiatry 1993;150:1063-1069.

-3 Shimabukuro J, Awata S, Matsuoka H: Behavioral and psychological symptoms of dementia characteristic of mild Alzheimer patients. Psychiatry Clin Neurosci 2005;59:274-279.

4 Mega MS, Cummings JL, Fiorello T, Gornbein J: The spectrum of behavioral changes in Alzheimer's disease. Neurology 1996;46:130-135.

5 Cummings JL, Mackell J, Kaufer D: Behavioral effects of current Alzheimer's disease treatments: a descriptive review. Alzheimers Dement 2008;4:49-60.

6 Coen RF, Swanwick GR, O’Boyle CA, Coakley D: Behaviour disturbance and other predictors of carer burden in Alzheimer's disease. Int J Geriatr Psychiatry 1997;12:331-336.

7 Steele C, Rovner B, Chase GA, Folstein M: Psychiatric symptoms and nursing home placement of patients with Alzheimer's disease. Am J Psychiatry 1990;147:1049-1051.

-8 Samochocki M, Höffle A, Fehrenbacher A, Jostock R, Ludwig J, Christner C, Radina M, Zerlin M, Ullmer C, Pereira $\mathrm{EF}$, Lübbert $\mathrm{H}$, Albuquerque EX, Maelicke A: Galantamine is an allosterically potentiating ligand of neuronal nicotinic but not of muscarinic acetylcholine receptors. J Pharmacol Exp Ther 2003;305:1024-1036.

-9 Kavanagh S, Gaudig M, Van Baelen B, Adami M, Delgado A, Guzman C, Jedenius E, Schäuble B: Galantamine and behavior in Alzheimer disease: analysis of four trials. Acta Neurol Scand 2011;124:302-308.

10 McKhann GM, Knopman DS, Chertkow H, Hyman BT, Jack CR Jr, Kawas CH, Klunk WE, Koroshetz WJ, Manly JJ, Mayeux R, Mohs RC, Morris JC, Rossor MN, Scheltens P, Carrillo MC, Thies B, Weintraub S, Phelps CH: The diagnosis of dementia due to Alzheimer's disease: recommendations from the National Institute on AgingAlzheimer's Association workgroups on diagnostic guidelines for Alzheimer's disease. Alzheimers Dement 2011;7:263-269.

11 Albert MS, DeKosky ST, Dickson D, Dubois B, Feldman HH, Fox NC, Gamst A, Holtzman DM, Jagust WJ, Petersen RC, Snyder PJ, Carrillo MC, Thies B, Phelps CH: The diagnosis of mild cognitive impairment due to Alzheimer's disease: recommendations from the National Institute on Aging-Alzheimer's Association workgroups on diagnostic guidelines for Alzheimer's disease. Alzheimers Dement 2011;7:270-279.

12 Matsumoto N, Ikeda M, Fukuhara R, Hyodo T, Ishikawa T, Mori T, Toyota Y, Matsumoto T, Adachi H, Shinagawa S, Hokoishi K, Tanabe H, Hirono N: Validity and reliability of the Japanese version of the Neuropsychiatric Inventory Caregiver Distress Scale (NPI D) and the Neuropsychiatric Inventory Brief Questionnaire Form (NPI-Q). No To Shinkei 2006;58:785-790.

13 Cummings JL, Mega M, Gray K, Rosenberg-Thompson S, Carusi DA, Gornbein J: The Neuropsychiatric Inventory: comprehensive assessment of psychopathology in dementia. Neurology 1994;44:2308-2314. 
14 Kaufer DI, Cummings JL, Ketched P, Smith V, MacMillan A, Shelley T, Lopez OL, DeKosky ST: Validation of the NPI-Q, a brief clinical form of the neuropsychiatric inventory. J Neuropsychiatry Clin Neurosci 2000;12:233239.

15 Fernández M, Gobartt AL, Balañá M; COOPERA Study Group: Behavioural symptoms in patients with Alzheimer's disease and their association with cognitive impairment. BMC Neurol 2010;10:87.

16 Swearer JM, Drachman DA, O’Donnell BF, Mitchell AL: Troublesome and disruptive behaviors in dementia. Relationships to diagnosis and disease severity. J Am Geriatr Soc 1988;36:784-790.

17 González-Salvador T, Lyketsos CG, Baker A, Hovanec L, Roques C, Brandt J, Steele C: Quality of life in dementia patients in long-term care. Int J Geriatr Psychiatry 2000;15:181-189.

18 Cummings JL, Back C: The cholinergic hypothesis of neuropsychiatric symptoms in Alzheimer's disease. Am J Geriatr Psychiatry 1998;6(2 suppl 1):S64-S78.

19 Tariot PN, Solomon PR, Morris JC, Kershaw P, Lilienfeld S, Ding CA: 5-Month, randomized, placebo-controlled trial of galantamine in AD. The Galantamine USA-10 Study Group. Neurology 2000;54:2269-2276.

-20 Brodaty H, Corey-Bloom J, Potocnik FC, Truyen L, Gold M, Damaraju CR: Galantamine prolonged-release formulation in the treatment of mild to moderate Alzheimer's disease. Dement Geriatr Cogn Disord 2005;20: 120-132.

21 Erkinjuntti T, Kurz A, Gauthier S, Bullock R, Lilienfeld S, Damaraju CV: Efficacy of galantamine in probable vascular dementia and Alzheimer's disease combined with cerebrovascular disease: a randomised trial. Lancet 2002;359:1283-1290.

22 Raskind MA, Peskind ER, Wessel T, Yuan W; the Galantamine USA-1 Study Group: Galantamine in AD: a 6-month, randomized, placebo-controlled trial with a 6-month extension. Neurology 2000;54:2261-2268.

23 Wilcock GK, Lilienfeld S, Gaens E; the Galantamine International-1 Study Group: Efficacy and safety of galantamine in patients with mild to moderate Alzheimer's disease: a multicentre randomized controlled trial. BMJ 2000;321:1445-1448.

24 Herrmann N, Rabheru K, Wang J, Binder C: Galantamine treatment of problematic behavior in Alzheimer disease: post-hoc analysis of pooled data from three large trials. Am J Geriatr Psychiatry 2005;13:527-534.

-25 Rockwood K, Mintzer J, Truyen L, Wessel T, Wilkinson D: Effects of a flexible galantamine dose in Alzheimer's disease: a randomized, controlled trial. J Neurol Neurosurg Psychiatry 2001;71:589-595.

-26 Martin-Ruiz CM, Court JA, Molnar E: $\alpha-4$ but not $\alpha-3$ and $\alpha-7$ nicotinic acetylcholine receptor subunits are lost from the temporal cortex in Alzheimer's disease. J Neurochem 1999;73:1635-1640.

27 Santos MD, Alkondon M, Pereira EF, Aracava Y, Eisenberg HM, Maelicke A, Albuquerque EX: The nicotinic allosteric potentiating ligand galantamine facilitates synaptic transmission in the mammalian central nervous system. Mol Pharmacol 2002;61:1222-1234.

28 Giacobini E: Cholinesterase inhibitors: from the Calabar bean to Alzheimer therapy; in Giacobini E (ed): Cholinesterases and Cholinesterase Inhibitors. London, Martin Dunitz, 2000, pp 181-226.

29 Ago Y, Koda K, Takuma K, Matsuda T: Pharmacological aspects of the acetylcholinesterase inhibitor galantamine. J Pharmacol Sci 2011;116:6-17.

-30 Wilkinson DG, Howe I; the GAL-GBR-1 Study Group: Switching from donepezil to galantamine: a double-blind study of two wash-out periods. Int J Geriatr Psychiatry 2005;20:489-491.

-31 Rasmusen L, Yan B, Robillard A, Dunbar F: Effects of washout and dose-escalation periods on the efficacy, safety, and tolerability of galantamine in patients previously treated with donepezil: ongoing clinical trials. Clin Ther 2001;23(suppl A):A25-A30.

-32 Edwards K, Therriault O’Connor J, Gorman C: Switching from donepezil or rivastigmine to galantamine in clinical practice. J Am Geriatr Soc 2004;52:1965.

-33 Maelicke A: Pharmacokinetic rationale for switching from donepezil to galantamine. Clin Ther 2001;23(suppl A):A8-A12.

34 Richarz U, Gaudig M, Rettig K, Schauble B: Galantamine treatment in outpatients with mild Alzheimer's disease. Acta Neurol Scand 2014;129:382-392. 